Draft VERsion MAY 24, 2022

Preprint typeset using $\mathrm{IAT}_{\mathrm{E}} \mathrm{X}$ style emulateapj v. 5/2/11

\title{
A RADIAL VELOCITY TEST FOR SUPERMASSIVE BLACK HOLE BINARIES AS AN EXPLANATION FOR BROAD, DOUBLE-PEAKED EMISSION LINES IN ACTIVE GALACTIC NUCLEI
}

\author{
Jia Liu ${ }^{1}$, Michael Eracleous ${ }^{2}$, And Jules P. Halpern ${ }^{1}$ \\ Draft version May 24, 2022
}

\begin{abstract}
One of the proposed explanations for the broad, double-peaked Balmer emission lines observed in the spectra of some active galactic nuclei (AGNs) is that they are associated with sub-parsec supermassive black hole $(\mathrm{SMBH})$ binaries. Here, we test the binary broad-line region hypothesis through several decades of monitoring of the velocity structure of double-peaked $\mathrm{H} \alpha$ emission lines in 13 low-redshift, mostly radio-loud AGNs. This is a much larger set of objects compared to an earlier test by Eracleous et al. (1997) and we use much longer time series for the three objects studied in that paper. Although systematic changes in radial velocity can be traced in many of their lines, they are demonstrably not like those of a spectroscopic binary in a circular orbit. Any spectroscopic binary period must therefore be much longer than the span of the monitoring (assuming a circular orbit), which in turn would require black hole masses that exceed by 1-2 orders of magnitude the values obtained for these objects using techniques such as reverberation mapping and stellar velocity dispersion. Moreover, the response of the double-peaked Balmer line profiles to fluctuations of the ionizing continuum and the shape of the Ly $\alpha$ profiles are incompatible with a SMBH binary. The binary broad-line region hypothesis is therefore disfavored. Other processes evidently shape these line profiles and cause the long-term velocity variations of the double peaks.

Subject headings: line: profiles - galaxies: active - galaxies: individual (1E 0450-1817, 3C 227, 3C 332, 3C 390.3, 3C 59, Arp 102B, CBS 74, Mrk 668, Pictor A, PKS 0235+023, PKS 0921-213, PKS 1020-103, PKS 1739+18) - quasars: emission lines
\end{abstract}

\section{INTRODUCTION}

Supermassive black hole (SMBH) binaries are thought to be a common, if not inevitable, outcome of the mergerdriven evolution of galaxies (Begelman et al. 1980; Volonteri et al. 2003, Di Matteo et al. 2005, Hopkins et al. 2006 e.g.). In the scenario described by Begelman et al. (1980), after two galaxies merge, their central BHs sink into the merger core through dynamical friction on a time scale of $\sim 10^{8}$ years. The loosely bound binaries $(\sim \mathrm{kpc}$ scale) later tighten to $\sim 1$ pc scale through the scattering of nuclear stars, until the "loss cone" 3 is depleted. Thereafter, gravitational wave driven angular momentum loss will not be significant until the separation is $10^{-2}-10^{-3} \mathrm{pc}$. The difficulty in shrinking the orbit after it reaches $\sim 1 \mathrm{pc}$ is the so-called "final parsec problem". Without an efficient mechanism to remove angular momentum, SMBH binaries can stall at the pc scale for longer than the Hubble time. More recent calculations invoking interactions of the SMBH binary with a gaseous reservoir (e.g., Armitage \& Natarajan 2002, Escala et al. 2004 Dotti et al. 2007, Hayasaki et al. 2007; Dotti et al. 2009 Cuadra et al. 2009; Lodato et al. 2009) or more realistic stellar dynamical models (such as non-spherical or rotating galaxies; e.g., Yu 2002, Merritt \& Poon 2004,

\footnotetext{
${ }^{1}$ Astronomy Department, Columbia University, 550 West 120th Street, New York, NY 10027, U.S.A.

2 Department of Astronomy and Institute for Gravitation and The Cosmos, The Pennsylvania State University, 525 Davey Lab, University Park, PA 16802, U.S.A.

${ }^{3}$ Stars residing in this cone in phase space have the right combination of positions and momenta to interact with the binary and eventually take away the angular momentum through gravitational slingshots.
}

Khan et al. 2013) suggest that SMBH binaries can evolve towards a merger quickly because they lose angular momentum efficiently.

Finding sub-parsec scale SMBH binaries (or confirming their absence) is crucial in our understanding of galaxy evolution (see recent reviews by Popović 2012. Schnittman 2013). However, there is no direct evidence for existence of such close SMBH binaries, although a number of candidates have been reported. Due to their close separation, we have little chance to spatially resolve the two BHs. There is only one close SBHB candidate that has been imaged with radio interferometers, CSO $0402+379$, with a separation of 7 pc (Maness et al. 2004, Rodriguez et al. 2006, 2009). All other current candidates have been suggested on the basis of indirect evidence.

Most of the proposed electromagnetic signatures of SMBH involve either radio jets or an accretion disk. For example, X-shaped radio jets seen in some galaxies are thought to reflect different spin directions of the two BHs (Merritt \& Ekers 2002, Zier \& Biermann 2002). Periodic flares in the light curve, such as the ones seen in OJ 287 approximately every 12 years (Sillanpaa et al. 1988 Lehto \& Valtonen 1996, Valtonen et. al. 2006), led the authors to propose a secondary BH plunging through the accretion disk of the primary BH. The nearby Seyfert galaxy NGC 4151 is a much more tentative case, suggested by Bon et al. (2012) on the basis of variability of its broad Balmer line profiles. Finally, thanks to the recent large sky surveys such as the Sloan Digital Sky Survey ${ }^{4}$ (SDSS), large systematic searches for spectro-

\footnotetext{
4 http://www.sdss.org/
} 
scopic signatures have become possible (e.g. Tsalmantza et al. 2011; Eracleous et al. 2012; Liu et al. 2013; Shen et al. 2013; Decarli et al. 2013), namely, single or doublepeaked emission lines, displaced from the host galaxy rest frame, and taken to indicate orbital motion in a SMBH binary (see Begelman et al. 1980; Gaskell 1983, 1996).

In this work we study the spectroscopic variability of 13 active galactic nuclei (AGNs) whose optical spectra display two displaced broad-line (BL) peaks, the so-called "double-peaked emitters" 1 (see examples in Figure 1).We concentrate on testing the SMBH binary interpretation for these line profiles. In our working scenario, each of the two BHs in the binary has an associated BL region, with the result that the orbital motion of the binary imparts a velocity separation between the lines from the two distinct BL regions (see the heuristic model and illustrations in Shen \& Loeb 2010). The separation of the peaks or shoulders in the observed line profiles suggests orbital separations of order $0.1 \mathrm{pc}$ and orbital periods of order a few centuries, assuming $\mathrm{BH}$ masses of order $10^{8} \mathrm{M}_{\odot}$ (see, for example, Equations 1 and 2 in Eracleous et al. 2012).

In addition to the reasons mentioned above, testing the SMBH binary hypothesis for double-peaked emitters helps answer the question of the origin of these line profiles. Several scenarios have been suggested in the literature, including SMBH binaries and emission from the outer parts of the accretion disk around a single BH (e.g., Chen et al. 1989, Chen \& Halpern 1989, Eracleous \& Halpern 1994; Strateva et al. 2003). The accretion disk interpretation has passed many observational tests and does not suffer from many drawbacks, unlike the other candidate explanations (a summary and critique of the scenarios can be found in Eracleous \& Halpern 2003, and Eracleous et al. 2009). The analysis we present here provides the most comprehensive test of the SBHB hypothesis to date. The combination of this test and several other observations and physical arguments that we summarize in Section 4 render the binary black hole hypothesis an unlikely candidate for explaining double-peaked emission lines.

As long-term monitoring is critical for finding evidence of orbital motion in the variations of the double-peaked line profiles, we collect in this paper measurements of spectra of the these objects from the literature, dating back to the 1970s. We supplement the published data with measurements we make from newly acquired spectra and from spectra retrieved from the SDSS spectroscopic archive. Using all the available data, we search for changes in the radial velocities of the displaced peaks or shoulders that can be attributed to Keplerian motion of two BHs, following the method of Eracleous et al. (1997). The collection of 13 objects we study here is substantially larger than the three objects studied by Eracleous et al. (1997). Those three objects, Arp 102B, 3C 390.3, and 3C 332, are included in our present collection but their time series are now 2, 1.5, and 1.3 times longer, respectively. As a result, we obtain more stringent constraints

5 The line profiles do not always show two clearly separated peaks. In some cases the profiles are flat-topped or show two shoulders. Nonetheless, there is substantial historical precedent of using this term to describe these profile shapes collectively, therefore we adopt the term here as well.
TABLE 1

TARGET LIST

\begin{tabular}{|c|c|c|c|c|}
\hline Object & R.A. (J2000) & Decl. (J2000) & $z$ & Refs. \\
\hline $1 \mathrm{E} 0450-1817$ & 045235.91 & -181201.64 & 0.0590 & 1 \\
\hline $3 \mathrm{C} 227$ & 094745.15 & +072520.60 & 0.0858 & $2,3,12,13$ \\
\hline $3 \mathrm{C} 332$ & 161742.52 & +322234.01 & 0.1517 & 2,12 \\
\hline 3C 390.3 & 184208.99 & +794617.13 & 0.0562 & $2-7,11$ \\
\hline $3 \mathrm{C} 59$ & 020702.18 & +293045.99 & 0.1100 & 1 \\
\hline Arp 102B & 171914.49 & +485849.43 & 0.0245 & $2,10,14$ \\
\hline CBS 74 & 083225.35 & +370736.26 & 0.0919 & 1,12 \\
\hline Mrk 668 & 140700.39 & +2827 14.69 & 0.0766 & 2,10 \\
\hline Pictor A & 051949.72 & -454643.85 & 0.0340 & $1,8,9$ \\
\hline PKS 0235+023 & 023832.68 & +023349.67 & 0.2090 & 2 \\
\hline PKS 0921-213 & 092338.89 & -213547.13 & 0.0534 & 1 \\
\hline PKS 1020-103 & 102232.81 & -103744.37 & 0.1970 & 1 \\
\hline PKS $1739+18$ & 174206.95 & +182721.06 & 0.1860 & 1 \\
\hline References.- (1) & \multicolumn{4}{|c|}{ Lewis et al. (2010); (2) Gezari et al. (2007); (3) } \\
\hline Osterbrock et al. & \multirow{2}{*}{\multicolumn{4}{|c|}{$\begin{array}{l}(1976) ;(4) \text { Yee \& Oke }(1981) ;(5) \text { Shater et al. } \\
\text { vic (2012); (7) Barr et al. }(1980) ; \frac{1}{(8) \text { Danziger }}\end{array}$}} \\
\hline (1985); (6) Роро & & & & \\
\hline et al. (1977); (9) & Carswell et al. & \multicolumn{3}{|c|}{ (1984); (10) Shapovalova et al. } \\
\hline (2013); (11) Gask & ell $(1996) ;(12)$ & \multicolumn{3}{|c|}{ (13) Netzer (1982); } \\
\hline
\end{tabular}

on the orbital parameters of putative binaries in these objects.

In Section 2, we describe the published data as well as our new observations and data reduction. In Section 3. we present the analysis of all available radial velocity measurements, and fit sinusoidal models to the velocity curves. We discuss the implications in Section 4, where we also present additional arguments against the SMBH binary hypothesis based on a variety of other observations. We summarize our findings and present our conclusions in Section 5 .

\section{OBSERVATIONS AND DATA REDUCTION}

We selected the 13 AGNs listed in Table 1 and compiled data from Eracleous et al. (1997), Gezari et al. (2007), and Lewis et al. (2010). The observations of the 13 objects by these authors span $\approx 20$ years, from approximately 1985 to 2005 . Their spectra were obtained using various telescopes of $1-\mathrm{m}$ to $9-\mathrm{m}$ aperture. We refer readers to these papers for detailed description of the observations and data reduction. We also retrieved additional historical spectra from the literature as well as from the SDSS. The sources of spectra for each object are listed in the last column of Table 1.

For four of the targets, we carried out additional observations spanning the years 2009-2013. We obtained our spectra using the MDM Observatory Hiltner $2.4 \mathrm{~m}$ and McGraw-Hill $1.3 \mathrm{~m}$ telescopes. Spectra were taken with either the Boller and Chivens CCD Spectrograph (CCDS) or with the Modular Spectrograph (Modspec). CCDS observations were obtained with a $150 \mathrm{~mm}^{-1}$ grating and a $1^{\prime \prime}$ slit, giving $7.6 \AA$ resolution. Modspec observations used a $600 \mathrm{~mm}^{-1}$ grating and a $1^{\prime \prime}$ slit, with $3.4 \AA$ resolution. One or multiple $900-1800 \mathrm{~s}$ exposures were taken for each target. The log of observations is given in Table 2 .

We reduced and calibrated the new spectra using the Image Reduction and Analysis Facility 7 (IRAF). For each object, the two-dimensional images were first bias and

\footnotetext{
6 http://mdm.kpno.noao.edu/

7 http://iraf.noao.edu/
} 
TABLE 2

MDM OBSERVATION LOG

\begin{tabular}{lllc}
\hline Object & Date (UT) & Instrument & Exposure (s) \\
\hline 3C 227 & 2009 Dec 16 & $2.4 \mathrm{~m}$ CCDS & 1200 \\
& 2011 Nov 26 & $1.3 \mathrm{~m}$ CCDS & $3 \times 1200$ \\
& 2013 May 04 & $2.4 \mathrm{~m}$ Modspec & 1800 \\
3C 390.3 & 2013 May 03 & $2.4 \mathrm{~m}$ Modspec & 1800 \\
Arp 102B & 2013 May 04 & $2.4 \mathrm{~m}$ Modspec & 900 \\
Mrk 668 & 2013 May 03 & $2.4 \mathrm{~m}$ Modspec & 1800 \\
\hline
\end{tabular}

flat-field corrected. Then the one-dimensional spectra were extracted using the apall routine. Wavelength calibration was done using comparison lamps of $\mathrm{Ar}, \mathrm{Hg}-\mathrm{Ne}$, and $\mathrm{Xe}$ with the identify, reidentify, and dispcor routines. The flux scale was calibrated using the spectra of standard stars by applying the standard, sensfunc, and calibrate routines. Finally, we correct the atmospheric B-band absorption for 3C 390.3 using the standard star Feige 34. Besides our follow-up observations, we also obtained four spectra from SDSS DR10 (see Table 1). Our reduced spectra and SDSS spectra are shown in Figure 1.

\section{ANALYSIS}

First, we follow the method described in Section 3.4 of Lewis et al. (2010) to measure the velocity of the shifted peaks of the $\mathrm{H} \alpha$ BLs for all spectra for which such measurements have not already been made. We then fit sinusoidal curves to the red and blue velocity peaks simultaneously, and obtain the best-fit and the minimum orbital period for each object allowed by the secular curvature of the radial velocity curve.

\subsection{Line Profiles}

For each spectrum, we measure the velocities using the IRAF splot routine. At the region of each peak, we mark two continuum points and fit a single Gaussian line profile. However, when the peak shapes are not Gaussian (for example, the flat-topped red peak of Mrk 668), the actual position of the peak can be quite uncertain. Hence, we perform multiple measurements using various continuum points and take the average to be the center. We also take half of the difference between our measured minimum and maximum peak velocities, or $100 \mathrm{~km} \mathrm{~s}^{-1}$ to be the error, whichever is larger. The $\mathrm{H} \alpha$ narrow line (NL) is taken to be at zero velocity. The velocity of a shifted peak of the $\mathrm{H} \alpha \mathrm{BL}$ is calculated as $v=\left(\Delta \lambda / \lambda_{\mathrm{NL}}\right) c$, where $\Delta \lambda=\lambda_{\mathrm{BL}}-\lambda_{\mathrm{NL}}$ and $c$ is the speed of light.

For observations where we do not have access to the digital data (i.e., references $3-11$ in Table 1), we convert the relevant published figure using Plot Digitizer ${ }^{8}$ and estimate the velocity of the shifted peaks by eye. These estimates are certainly not as accurate as measurements done on numerical data, but give us valuable historical information on line shifts. However, only figures from Osterbrock et al. (1976), Barr et al. (1980), and Shapovalova et al. (2013) have high enough resolution that we can measure the velocity confidently. Gaskell (1996), Gezari et al. (2007), Lewis et al. (2010), Popovic (2012), and $\overline{\text { Popovic et al. }}(2014)$ presented velocity shift measurements, which we use directly in our analysis. These

\footnotetext{
8 http://plotdigitizer.sourceforge.net/
}

TABLE 3

Newly Measured velocities

\begin{tabular}{llccc}
\hline Target & Date (UT) & $\begin{array}{c}U_{1} \text { (blue) } \\
\left(10^{3} \mathrm{~km} \mathrm{~s}^{-1}\right)\end{array}$ & $\begin{array}{c}U_{2}(\mathrm{red}) \\
\left(10^{3} \mathrm{~km} \mathrm{~s}^{-1}\right)\end{array}$ & Refs. \\
\hline 3C 227 & 1974 May/Dec & $-2.04 \pm 0.10$ & $\ldots$ & 1 \\
& 2003 Mar & $\ldots$ & $0.95 \pm 0.10$ & 5 \\
& 2009 Dec 16 & $-1.5 \pm 0.10$ & $\ldots$ & 2 \\
& 2011 Nov 26 & $-0.95 \pm 0.10$ & $\ldots$ & 2 \\
& 2013 May 04 & $-0.47 \pm 0.10$ & $0.96 \pm 0.10$ & 2 \\
3C 390.3 & 1974 May/Jun & $-3.22 \pm 0.64$ & $4.67 \pm 0.69$ & 1 \\
& 1975 Jul & $-4.00 \pm 0.50$ & $\ldots$ & 3 \\
& 2013 May 04 & $-4.18 \pm 0.29$ & $6.34 \pm 0.10$ & 2 \\
Arp 102B & 1998 Jul & $-5.47 \pm 0.10$ & $\ldots$ & 4 \\
& 2003 Mar & $-5.47 \pm 0.10$ & $\ldots$ & 4 \\
& 2006 Aug & $-5.56 \pm 0.10$ & $\ldots$ & 4 \\
Mrk 668 & 2013 May 04 & $-5.31 \pm 0.10$ & $6.74 \pm 0.10$ & 2 \\
& 2006 Feb 27 & $-4.48 \pm 0.10$ & $3.79 \pm 0.10$ & 5 \\
CBS 74 & 2013 May 03 & $\ldots$ & $3.83 \pm 0.10$ & 2 \\
3C 332 & 2002 Feb 06 & $-2.15 \pm 0.10$ & $3.86 \pm 0.10$ & 5 \\
2004 Aug 22 & $-5.88 \pm 0.10$ & $7.90 \pm 0.10$ & 5 \\
\hline
\end{tabular}

References.- (1) Osterbrock et al. (1976); (2) This work; (3) Barr et al. (1980); (4) Shapovalova et al. (2013); (5) SDSS.

authors used different methods to determine the peak location. Gaskell (1996) used the Pogson method, which is very similar to fitting a Gaussian to the region of the line profile close to the peak; Gezari et al. (2007) measured the flux-weighted centroid of the top $10 \%$ of the peak; Lewis et al. (2010) and Popović et al. (2014) fitted a Gaussian profile to the peak; Popovic (2012) used the parameters resulting from a disk model. The different measurements are fairly consistent when examined by eye, except for the noticeable difference between the redshifted velocity component in 3C390.3 measured by Gezari et al. (2007) and that by Popović (2012). This is likely due to their different methods of removing the NLs. For consistency, we exclude the data of Popović (2012) from the periodicity test in Section 3.2. We also note that Gaskell (1996) measured the blue peak of $\mathrm{H} \beta$ instead of $\mathrm{H} \alpha$.

The measured velocities from the spectra obtained in this work and from the digitized figures are listed in Table 3. Radial velocity curves for each object are shown in Figure 2 and we examine them for the signature of orbital motion next. These measurements extend the previously published spectroscopic time series for Arp 102B, 3C 390.3, 3C 227, and Mrk 668 by factors of 1.7, 1.25, 2.7 , and 1,6 respectively, which substantially influences the binary orbital parameters we derive below.

\subsection{Tests for Orbital Motion}

To derive constraints on the total mass of a hypothesized SMBH binary, we follow the method of Eracleous et al. (1997). In summary, we adopt a circular orbit model and fit the observed radial velocities $u_{1}$ and $u_{2}$ of the two peaks with the following model

$$
\begin{aligned}
& u_{1}(t)=-\left(v_{1} \sin i\right) \sin \left[\frac{2 \pi}{P}\left(t-t_{0}\right)\right] \text { and } \\
& u_{2}(t)=\left(v_{2} \sin i\right) \sin \left[\frac{2 \pi}{P}\left(t-t_{0}\right)\right],
\end{aligned}
$$

where $v_{1}, v_{2}$ are the true (i.e. tangential) orbital velocities, $i$ is the inclination angle, $P$ is the period, and $t_{0}$ is the time at which the two peaks coincide at zero ra- 

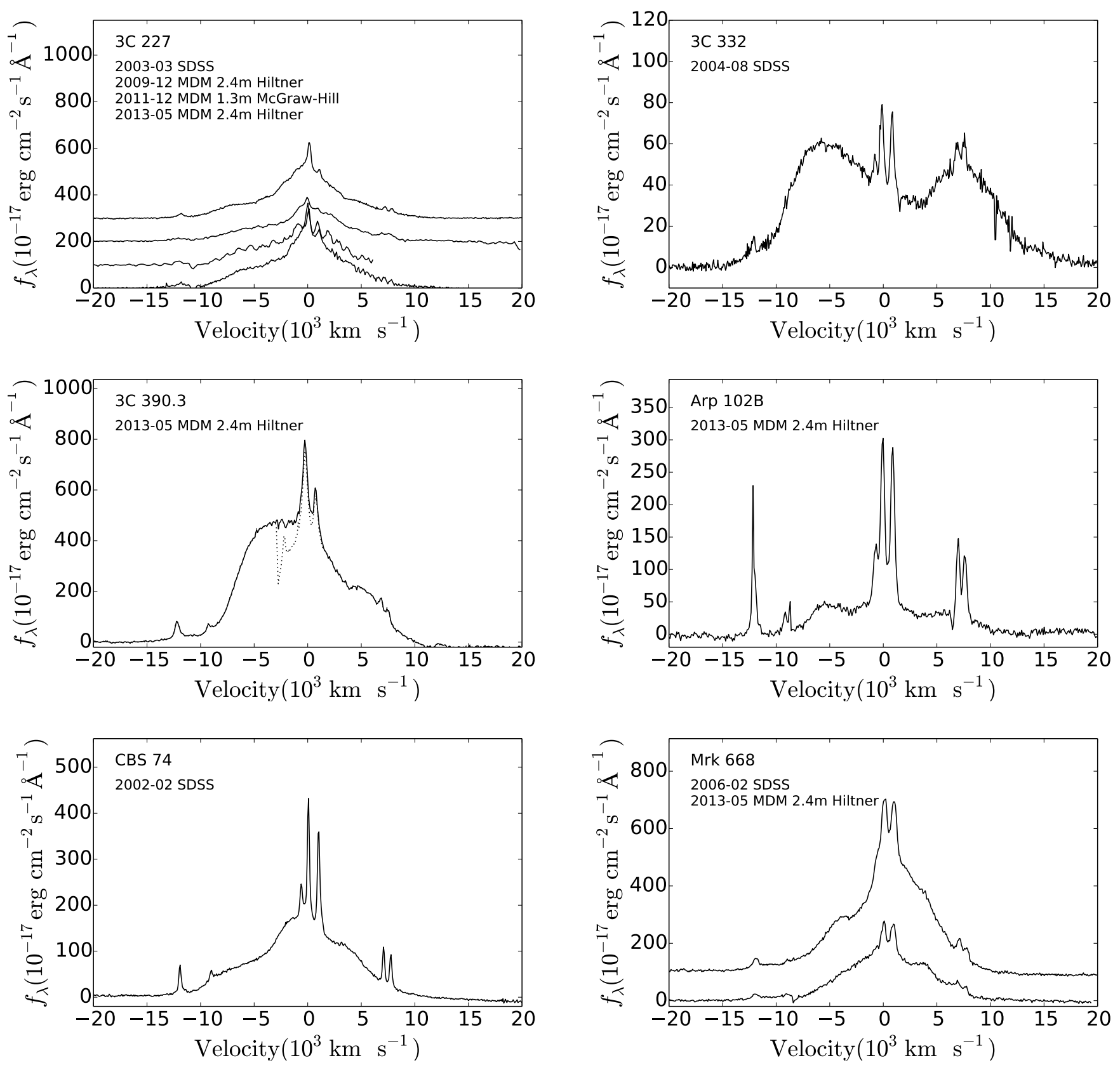

Fig. 1. - Six H $\alpha$ spectra obtained during 2009-2013 at MDM Observatory, and four spectra from the SDSS. The dotted line in 3C 390.3 shows the original spectrum before atmospheric B-band correction. Vertical offsets have been applied for clarity.

dial velocity (conjunction). The assumption of circular orbits is justified by the results of stellar dynamics simulations that follow the evolution of the binary following the merger of the two parent galaxies. As a rule, for the eccentricity to grow appreciably it has to start out with values, $e \gtrsim 0.5$, and the binary mass ratio should also be large, $q=M_{1} / M_{2} \gtrsim 10$ (e.g., Quinlan 1996, Sesana et al. 2006. Wang et al. 2014, Vasiliev et al. 2015). As we note below, the velocity ratio of the two peaks in the double-peaked profiles of our sample imply small mass ratios, $q=1-2$, which would suppress the growth of eccentricity. Most relevant to the problem we are concerned with here are the N-body simulations of merging galaxies by Khan et al. (2013) and Holley-Bockelmann \& Khan (2015), which find that the eccentricity of the bi- nary quickly decays to zero for galaxies with no rotation or net prograde rotation. For galaxies with retrograde rotation, the eccentricity is quickly driven to unity and the binary merges as soon as it hardens.

To find the best-fitting model parameters, we use two schemes. In the first scheme, we minimize the $\chi^{2}$ statistic, given by

$$
\chi^{2}=\sum_{i=1}^{N_{1}}\left(\frac{U_{1}^{i}-u_{1}^{i}}{\sigma_{1}^{i}}\right)^{2}+\sum_{i=1}^{N_{2}}\left(\frac{U_{2}^{i}-u_{2}^{i}}{\sigma_{2}^{i}}\right)^{2}
$$

where $U_{1}, U_{2}$ are the observed velocities, $u_{1}, u_{2}$ are models from equation 1 , and $N_{1}, N_{2}$ are the number of data points for the two velocity peaks. Note that uncertainties in velocity mainly come from line profile changes, 
possibly due to either reverberation or dynamical effects (e.g., Barth et al. 2015, Eracleous et al. 1997, respectively), rather than measurement errors such as those in Table 3. Therefore, we set the error bar, $\sigma$, to be the standard deviation of multiple measurements within a year. When there is only one observation available in a year, we use the average error from other epochs where multiple measurements of the same object are available. In practice, we scan through the three-dimensional parameter space $\left(t_{0}, v_{1} \sin i, v_{2} \sin i\right)$ for $P \in\left[5,10^{4}\right]$ years with 100 logarithmically spaced values of $P$. The bestfitting model parameters obtained by this scheme are summarized in Table 4, where we also include the reduced $\chi^{2}$ values for the best fits, $\chi_{\nu, \min }^{2}$, and the number of degrees of freedom. In several cases, we were not able to find the minimum $\chi^{2}$ in the range of periods that we tested because $\chi^{2}$ decreases monotonically up to the maximum period we consider. Therefore, we conclude that $P>10^{4}$ yr and we indicate this conclusion in Table 4. As one can see in Table 4. the values $\chi_{\nu, \min }^{2}$ are significantly larger than unity, indicating that the models do not provide a satisfactory description of the data. In the case of PKS 0235+023, the probability that the binary orbit model is a suitable model is 0.06 while in all other cases this probability is less than $8 \times 10^{-5}$. Even though a better fit may be possible for periods greater than $10^{4}$ yr for some objects, such periods lead to unreasonably high black hole masses, as we show later in this section.

In the second scheme we minimize the unweighted squared deviations of the model from the data. In other words, we minimize the statistic of equation (2) after setting $\sigma_{1,2}^{i}$ to unity for all data points. This scheme amounts to accepting that there will be some scatter in the individual measurements as a result of profile variability on times scales of order a few years. In Figure 5 we show the best-fitting models from this scheme as solid lines superposed on the data. The best-fitting model parameters are listed in Table 5 . The periods obtained by the unweighted least squares method are similar to those obtained by the $\chi^{2}$ method. We also note that the periods allowed for each object are much longer than the span of our monitoring program, rendering any "periodicity" found in this analysis unreliable. In column 7 of Table 5. we give the expected year of the next conjunction, according to the best-fitting model. At the time of conjunction, according to the binary model, the two peaks will overlap and the emission line profile will appear single-peaked and have its minimum width.

We also searched for periodic signals in both velocities and fluxes using the Lomb-Scargle periodogram (Lomb 1976, Scargle 1982 ${ }^{9}$ The Lomb-Scargle analysis is similar to the ordinary power spectrum, but particularly suitable for unevenly distributed data points. It also has the advantage of finding the phase automatically. Similar to what we found using sinusoidal fits, the periodogram did not find any significant period for our objects that is within the length of monitoring span.

We conclude that for the objects of interest in this work, either the periods are much longer than 20 years,

\footnotetext{
${ }^{9}$ For velocities, we use all the observations available except for the data points from Popović (2012). For fluxes, we only use the measurements by Gezari et al. (2007) and Lewis et al. (2010).
}

or mechanisms other than orbiting $\mathrm{BHs}$ are responsible for the emission-line velocity changes.

Notwithstanding the significant deviations of the observed radial velocity curves from the orbital models, we go on to explore the consequences of the orbital periods implied by the fits. Assuming the two BHs have masses $M_{1}$ and $M_{2}$, with a mass ratio $q=M_{1} / M_{2} \geq 1$, we obtain a lower limit on the total mass of the binary $M=M_{1}+M_{2}$ using the following relations

$$
M>4.7 \times 10^{8}(1+q)^{3}\left(\frac{P}{100 \mathrm{yr}}\right)\left(\frac{v_{1} \sin i}{5000 \mathrm{~km} \mathrm{~s}^{-1}}\right)^{3} \mathrm{M}_{\odot}
$$

or

$$
M>4.7 \times 10^{8}\left(\frac{1+q}{q}\right)^{3}\left(\frac{P}{100 \mathrm{yr}}\right)\left(\frac{v_{2} \sin i}{5000 \mathrm{~km} \mathrm{~s}^{-1}}\right)^{3} \mathrm{M}_{\odot} .
$$

(see Eracleous et al. 1997). The mass ratio is inferred from the best-fitting velocity amplitudes through $q=$ $v_{2} / v_{1}$. For objects with only one peak visible, even though it is not possible to estimate $q$ we can still put a constraint on the total mass since $q \geq 1$ by definition; therefore, $(1+q)^{3} \geq 8$ and $[(1+q) / q]^{3} \geq 1$. The values for total mass of the SMBH binary that we obtain by the this approach are listed in Tables 4 and 5 .

\section{DISCUSSION OF RESULTS}

There have been many similar attempts to find SMBH binaries using displaced broad peaks, but none have succeeded. Gaskell (1996) showed velocity shifts in the spectrum of $3 \mathrm{C} 390.3$ that could be fitted with a 300 year period. However, Eracleous et al. (1997) later rejected this interpretation, as they found that the motion in 3C 390.3 stopped after 1988 (which is also evident from our Figure 2). Lacking clear spectroscopic evidence for orbital motion in the velocity curves, the three objects chosen for study by Eracleous et al. (1997), 3C 390.3, Arp 102B, and $3 \mathrm{C} 332$, were found to require masses $>10^{10} M_{\odot}$ under the binary $\mathrm{BL}$ region hypothesis, and our extension of that monitoring has only increased those limits. Such masses are far in excess of those measured using direct methods such as reverberation mapping, from which Shapovalova et al. (2013) estimated Arp 102B to have $M=1.1 \times 10^{8} \mathrm{M}_{\odot}$, and Sergeev et al. (2011) estimated $3 \mathrm{C} 390.3$ to have $M=2.0 \times 10^{9} \mathrm{M}_{\odot}$, both much less than $10^{12} \mathrm{M}_{\odot}$ and $10^{14} \mathrm{M}_{\odot}$, respectively, obtained under the binary BL region assumption (see Tables 4 and 5 ).

$\mathrm{BH}$ mass estimates for several of the objects in this study were also made by Lewis \& Eracleous (2006) using the velocity dispersion in the Ca II infrared triplet and the correlation between $\mathrm{BH}$ mass and stellar velocity dispersion. The results range from $4 \times 10^{7} M_{\odot}$ for 1E 0450.3-1817, Pictor A, and PKS 0921-213, to $1.1 \times 10^{8} M_{\odot}$ for Arp $102 \mathrm{~B}$, and $5 \times 10^{8} M_{\odot}$ for $3 \mathrm{C} 390.3$. These are all a few orders of magnitude smaller than the masses derived under the binary BL region assumption (listed in Tables 4 and 5), a further indication that the latter hypothesis is untenable.

We offer the following additional arguments against the SMBH binary interpretation of double-peaked emission lines.

1. The profiles of the Ly $\alpha$ lines of double-peaked emit- 
TABLE 4

BEST-FIT BINARY ORBIT PARAMETERS FROM THE $\chi^{2}$ METHOD

\begin{tabular}{|c|c|c|c|c|c|c|}
\hline $\begin{array}{l}\text { Object } \\
(1)\end{array}$ & $\begin{array}{c}P \\
(\mathrm{yr}) \\
(2) \\
\end{array}$ & $\begin{array}{c}v_{1} \sin i \\
\left(10^{3} \mathrm{~km} \mathrm{~s}^{-1}\right) \\
(3)\end{array}$ & $\begin{array}{c}v_{2} \sin i \\
\left(10^{3} \mathrm{~km} \mathrm{~s}^{-1}\right) \\
(4)\end{array}$ & $\begin{array}{c}q \\
(5) \\
\end{array}$ & $\begin{array}{c}\log \left(M / M_{\odot}\right) \\
(6) \\
\end{array}$ & $\begin{array}{c}\chi_{\nu, \min }^{2}(\text { d.o.f. }) \\
(7)\end{array}$ \\
\hline 1E $0450.3-1817$ & 37 & 3.34 & 5.56 & 1.66 & 9.0 & $3.41(33)$ \\
\hline $3 \mathrm{C} 227$ & 171 & $\ldots$ & 2.29 & $\ldots$ & $>7.9$ & $6.42(23)$ \\
\hline 3C 332 & 158 & 8.4 & 10.66 & 1.27 & 10.6 & $5.77 \quad(59)$ \\
\hline 3C 390.3 & $>10^{4}$ & 30.63 & 37.10 & 1.21 & $>14.1$ & $7.66 \quad(99)$ \\
\hline $3 \mathrm{C} 59$ & 79 & 2.11 & 4.11 & 1.95 & 8.9 & $6.14 \quad(21)$ \\
\hline Arp 102B & $>10^{4}$ & 7.96 & 8.55 & 1.07 & $>12.2$ & $3.09(176)$ \\
\hline CBS 74 & 79 & 1.9 & 4.00 & 2.11 & 8.8 & $2.77 \quad(29)$ \\
\hline Mrk 668 & $>10^{4}$ & 72.57 & $\ldots$ & $\ldots$ & $>14.2$ & $9.92 \quad(21)$ \\
\hline Pictor A & 136 & 5.1 & 5.49 & 1.08 & 9.8 & $4.11 \quad(17)$ \\
\hline PKS $0235+023$ & 232 & $\ldots$ & 5.56 & $\ldots$ & $>9.2$ & $1.63(14)$ \\
\hline PKS 0921-213 & $>10^{4}$ & 16.59 & 16.64 & 1.00 & $>14.1$ & $2.47(24)$ \\
\hline PKS 1020-103 & $>10^{4}$ & 24.39 & 32.45 & 1.33 & $>13.8$ & $4.94 \quad(15)$ \\
\hline PKS $1739+18$ & 79 & 2.79 & 3.60 & 1.29 & 8.9 & $4.15 \quad(31)$ \\
\hline
\end{tabular}

Table Columns.- (1) object name, (2) best-fitting orbital period, (3) projected velocity amplitude of primary, (4) projected velocity amplitude of secondary, (5) mass ratio $\left(q=v_{2} / v_{1}\right)$, (6) total mass of binary implied by best-fitting model, (7) reduced $\chi^{2}$ corresponding to best fit and number of degrees of freedom.

TABLE 5

BEST-FIT BINARY ORBIT PARAMETERS FROM THE UNWEGHTED LEAST SQUARES METHOD

\begin{tabular}{|c|c|c|c|c|c|c|}
\hline Object & $\begin{array}{c}P \\
(\mathrm{yr}) \\
(2)\end{array}$ & $\begin{array}{c}v_{1} \sin i \\
\left(10^{3} \mathrm{~km} \mathrm{~s}^{-1}\right) \\
(3)\end{array}$ & $\begin{array}{c}v_{2} \sin i \\
\left(10^{3} \mathrm{~km} \mathrm{~s}^{-1}\right) \\
(4)\end{array}$ & $\begin{array}{c}q \\
(5)\end{array}$ & $\begin{array}{c}\log \left(M / M_{\odot}\right) \\
(6)\end{array}$ & $\begin{array}{l}t_{0} \\
(7)\end{array}$ \\
\hline 1E $0450.3-1817$ & 54 & 3.29 & 4.82 & 1.47 & 9.0 & 2037 \\
\hline $3 \mathrm{C} 227$ & 158 & $\ldots$ & 2.26 & $\ldots$ & $>7.8$ & 2097 \\
\hline 3C 332 & 136 & 7.56 & 10.23 & 1.35 & 10.5 & 2089 \\
\hline 3C 390.3 & $>10^{4}$ & 33.45 & 39.03 & 1.17 & $>14.2$ & $\ldots$ \\
\hline $3 \mathrm{C} 59$ & 79 & 2.37 & 4.16 & 1.76 & 8.9 & 2060 \\
\hline Arp 102B & $>10^{4}$ & 5.65 & 5.75 & 1.02 & $>11.7$ & $\ldots$ \\
\hline CBS 74 & 100 & 1.75 & 3.75 & 2.14 & 8.8 & 2069 \\
\hline Mrk 668 & $>10^{4}$ & 18.58 & $\ldots$ & 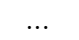 & $>12.4$ & $\ldots$ \\
\hline Pictor A & $>10^{4}$ & 8.96 & 9.35 & 1.04 & $>12.4$ & $\ldots$ \\
\hline PKS $0235+023$ & 171 & & 4.85 & & $>8.9$ & 2139 \\
\hline PKS 0921-213 & $>10^{4}$ & 6.99 & 7.01 & 1.00 & $>12.0$ & $\ldots$ \\
\hline PKS 1020-103 & $>10^{4}$ & 11.71 & 15.6 & 1.33 & $>12.9$ & $\ldots$ \\
\hline PKS 1739+18 & 100 & 2.66 & 3.48 & 1.31 & 8.9 & 2070 \\
\hline
\end{tabular}

Table Columns.- (1) object name, (2) best-fitting orbital period, (3) projected velocity amplitude of primary, (4) projected velocity amplitude of secondary, (5) mass ratio $\left(q=v_{2} / v_{1}\right)$, (6) total mass of binary implied by best-fitting model, (7) expected year of next conjunction (i.e., time of vanishing projected velocities).

ters, are not double-peaked. Instead they have a single peak that is located close to the systemic redshift, i.e., between the two peaks of the broad Balmer lines (Halpern et al. 1996, Eracleous et al. 2009). These argue strongly against any scenario that attributes the peaks of the Balmer lines to separate and physically distinct gaseous regions that move relative to each other.

2. In the case of 3C 390.3, we can use constraints on the inclination angle of the radio jet, assumed to be perpendicular to the orbital plane, to refine the constraint on the mass of a hypothesized SMBH binary: Eracleous et al. (1996) estimated that $i>19^{\circ}$ based on the observed superluminal motion in the radio jet. Applying this constraint raises the minimum mass of the binary to $\log \left(M_{\min } / M_{\odot}\right)=14.6$, which is rather implausible. Moreover, the binary separation in 3C 390.3 implied by the constraints is $28 \mathrm{pc}$. This is in con- tradiction with the results of reverberation mapping of $3 \mathrm{C} 390.3$ that show the two peaks to respond to variations of the continuum virtually simultaneously (within 3 days of each other, much shorter than the light crossing time corresponding to $>28$ pc; see Dietrich et al. 2012). Similarly, in the case of Arp 102B, the binary separation implied by the available constraints is $>6 \mathrm{pc}$. In contrast the entire broad $\mathrm{H} \alpha$ line responds to continuum changes within 22 days (Shapovalova et al. 2013) with a negligible lag between the blue and red sides (Popović et al. 2014), i.e., far from what one would expect for two widely-separated BL regions.

3. The working hypothesis on which the radial velocity test for a SMBH binary is based is that the two members of the binary are moving relative to the rest frame of the host galaxy and carrying with them the gas in the BL region (see for example, the models of Hayasaki et al. 2007; Cuadra et al. 2009, 
for a possible geometry). The width of the emission lines from the binary is dominated by the individual BL region rather than by the orbital velocities of the BH binary. The projected velocity separation between the two BLs should be considerably smaller than their widths based simply on Kepler's laws. This was pointed out by Chen et al. (1989, see their footnote 3 ) and more recently demonstrated by Shen \& Loeb (2010) using a heuristic model for the BL region. Thus, we should not expect the combined line profile from a SMBH binary to show two well separated peaks as is the case for several of the objects in our sample (e.g., Arp 102B, 3C 332, Pictor A, PKS 0921-21).

Finally, we note that some objects (3C 332, 3C 59, and CBS 74) do show interesting symmetric shifts between the red and the blue Balmer line peaks. However, peaks crossing at zero velocity, a signature we expect to see in binary motion, is not observed for any of these objects. A similar behavior is seen is NGC 1097, a doublepeaked emitter not included in this study (see Figure 5 of Schimoia et al. 2015). In NGC 1097 the separation between the two peaks of the broad $\mathrm{H} \alpha$ line changes in accordance with the integrated flux of the lines; as the peak separation fluctuates the two peaks never cross. A similar correlation can be discerned for 3C 390.3 by inspecting Figure 4 of Shapovalova et al. (2001). A short segment of the velocity curve of NGC 1097 could easily be interpreted as a segment of a sinusoid and attributed (incorrectly) to orbital motion of two BL regions.

There remains a possibility that the orbits of some SMBH binaries are eccentric, which would mean that the orbital models we have adopted for our analysis are not applicable. For a complete analysis, models of eccentric orbits should also be compared with the data. But even if such models were found to be viable and to yield reasonable BH masses, the additional arguments against the SMBH binary hypothesis presented above would remain valid.

In view of the above arguments, interpretations other than SMBH binaries should be considered for the double peaked Balmer lines. To fit the line profiles of Arp 102B and 3C 390.3, Chen \& Halpern (1989) proposed a thin disk illuminated by a thick hot inner torus, with only one $\mathrm{BH}$ present in the center. In this model, velocity shifts can be attributed to transient bright spots or other structures in the disk (e.g., Newman et al. 1997). Alternatively, slow, large amplitude variations in the illumina- tion of such a disk could be responsible for the apparent radial velocity changes of the Balmer line peaks (e.g., Schimoia et al. 2015, and references therein).

\section{SUMMARY, CONCLUSIONS, AND FUTURE PROSPECTS}

We searched for periodic radial velocity variations in the broad, double-peaked Balmer emission lines of 13 AGNs for which multi-decade monitoring observations are available. We obtained velocity measurements for these objects from multiple sources: Gezari et al. (2007), Lewis et al. (2010), Popović (2012), Popović et al. (2014), follow-up observations for four AGNs at the MDM Observatory in 2009-2013, four SDSS archival spectra, and digitized graphic spectra from the literature (see Table 1). We searched for periodic signals in both flux and velocity variations, but without success. We conclude that for these AGNs, any periods are significantly longer than our monitoring span, and/or mechanisms other than orbiting $\mathrm{BHs}$ are responsible for their double-peaked broad $\mathrm{H} \alpha$ lines and their line profile changes. We also presented additional arguments based on other observations that strongly disfavor the SMBH binary interpretation of broad, double-peaked emission lines.

By extension, newer studies of single displaced-peak emission lines in AGNs will have to take into account the possibility that the same processes will manifest themselves as radial velocity variations that are not directly related to orbital motion of $\mathrm{SMBH}$ binaries or recoiling SMBH mergers. This velocity "noise" complicates the search for kinematic evidence of SMBH motion in broad emission lines, placing more stringent observational requirements on the duration and coherence of periodic signals than have been attained so far. Potential sources of such noise include reverberation, as discussed by Barth et al. (2015), and other phenomena that occur on longer time scales (on the order of the dynamical time or longer; see illustration in Fig. 5 of Schimoia et al. 2015). It is, therefore, useful to continue monitoring the profiles of double-peaked Balmer emission lines on long time scales to check for non-monotonic changes of the velocities of the two peaks that would provide a further test of the SMBH binary hypothesis. If, for example, the trend of decreasing separation of the two peaks in some objects (e.g., 3C 332) reverses itself before the peaks cross at zero velocity (as in 3C 390.3), this would further weaken the case for SMBH binaries in double-peaked emitters.

This work was supported by grant AST-1211756 from the National Science Foundation. We acknowledge comments and suggestions from an anonymous referee.

\section{REFERENCES}

Armitage, P. J., \& Natarajan, P. 2002, ApJ, 567, L9

Barr, P., Pollard, G., Sanford, P. W., et al. 1980, MNRAS, 193, 549

Barth, A. J., Bennert, V. N., Canalizo, G., et al. 2015, ApJS, 217, 26

Begelman, M. C., Blandford, R. D., \& Rees, M. J. 1980, Nature, 287, 307

Bon, E., Jovanović, P., Marziani, P., et al. 2012, ApJ, 759, 118

Carswell, R. F., Baldwin, J. A., Atwood, B., \& Phillips, M. M. 1984, ApJ, 286, 464

Chen, K., \& Halpern, J. P. 1989, ApJ, 344, 115

Chen, K., Halpern, J. P., \& Filippenko, A. V. 1989, ApJ, 339, 742
Cuadra, J., Armitage, P. J., Alexander, R. D., \& Begelman, M. C. 2009, MNRAS, 393, 1423

Danziger, I. J., Fosbury, R. A. E., \& Penston, M. V. 1977, MNRAS, $179,41 \mathrm{P}$

Decarli, R., Dotti, M., Fumagalli, M., et al. 2013, MNRAS, 433, 1492

Di Matteo, T., Springel, V., \& Hernquist, L. 2005, Nature, 433, 604

Dietrich, M., Peterson, B. M., Grier, C. J., et al. 2012, ApJ, 757, 53

Dotti, M., Colpi, M., Haardt, F., \& Mayer, L. 2007, MNRAS, 379,956 

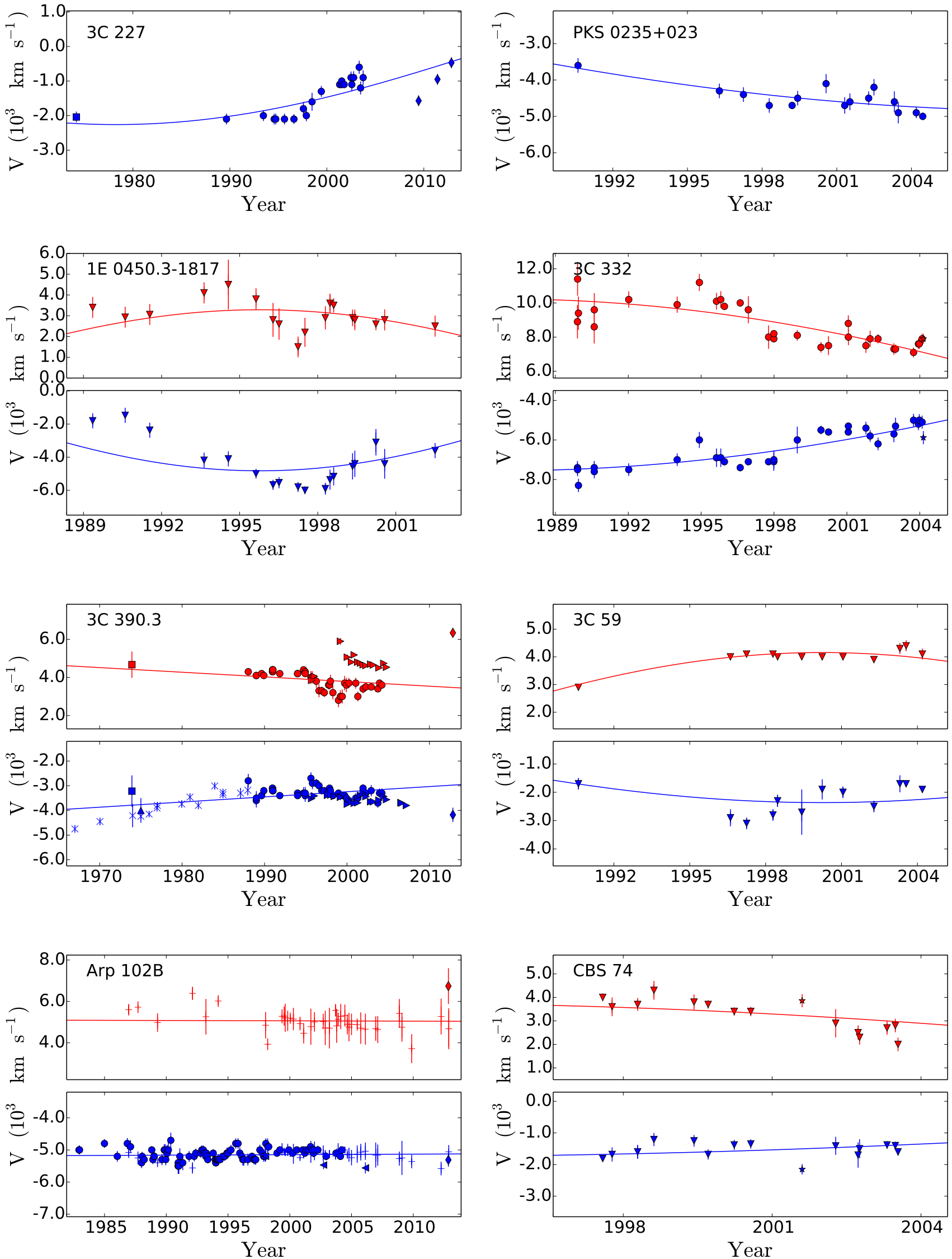

FIG. 2.- Velocities of the shifted peaks of the broad H $\alpha$ line and fitted curves. Circle: Gezari et al. (2007); Down-triangle: Lewis et al. (2010); Square: Osterbrock et al. (1976); Up-triangle: Barr et al. (1980); Diamond: This work; "x": Gaskell (1996); Left-triangle: Shapovalova et al. (2013); Right-triangle: Popović (2012); Plus: Popovic et al. (2014); Star: SDSS. Solid curves are the best-fit models from the unweighted least squares method (Table 5). Note that the velocity scale on the vertical axis has been expanded to show detail (i.e., it does not start at zero). 

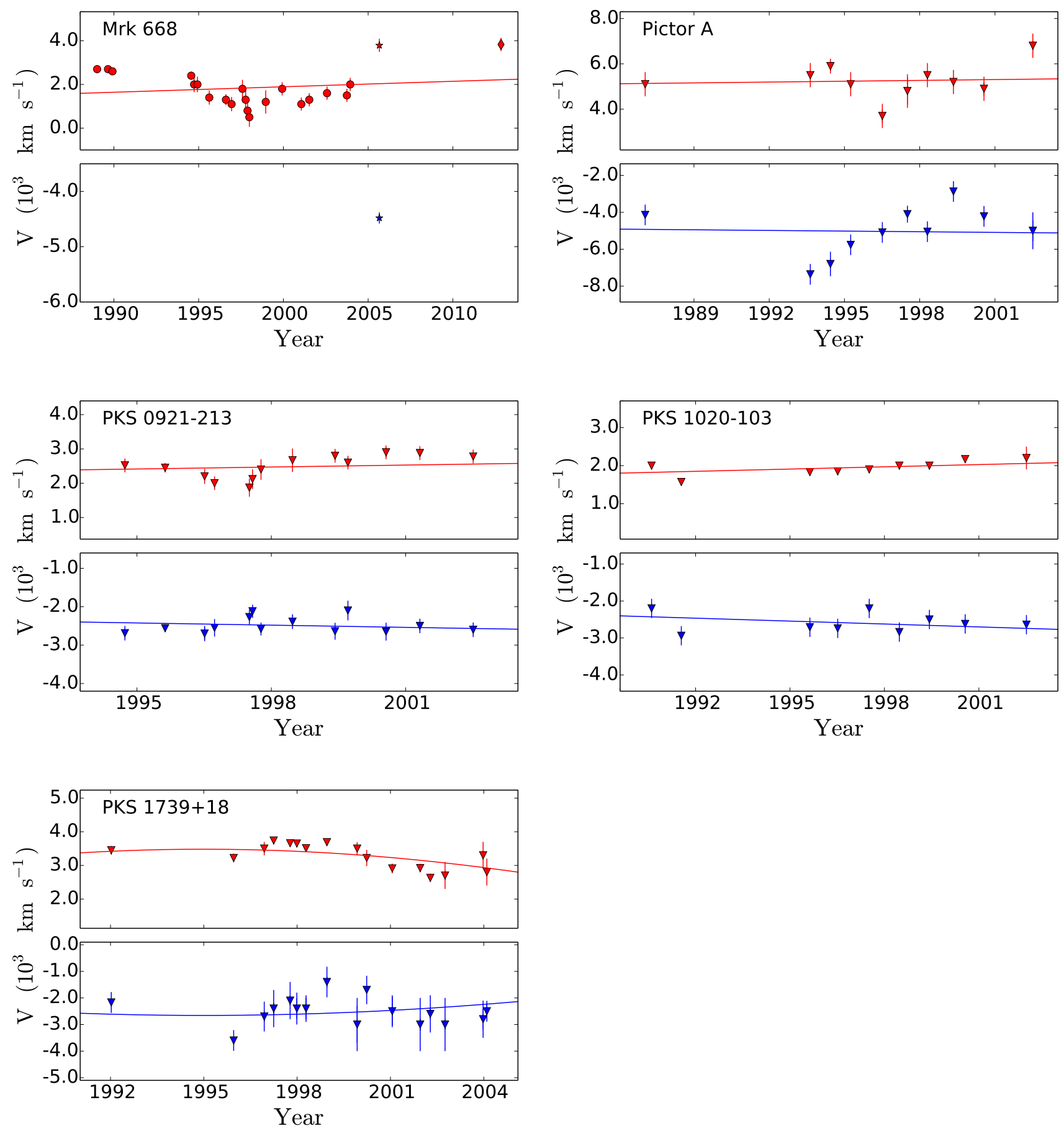

Fig. 2 (CONT.).- Velocities of the shifted peaks of the broad H $\alpha$ line and fitted curves. Circle: Gezari et al. (2007); Down-triangle: Lewis et al. (2010); Square: Osterbrock et al. (1976): Up-triangle: Barr et al. (1980): Diamond: This work; "x": Gaskell (1996); Left-triangle: Shapovalova et al. (2013); Right-triangle: Popović (2012); Plus: Popovic et al. (2014); Star: SDSS. Solid curves are the best-fit models from the unweighted least squares method (Table 5). Note that the velocity scale on the vertical axis has been expanded to show detail (i.e., it does not start at zero). 
Dotti, M., Ruszkowski, M., Paredi, L., et al. 2009, MNRAS, 396, 1640

Eracleous, M., Boroson, T. A., Halpern, J. P., \& Liu, J. 2012, ApJS, 201, 23

Eracleous, M., \& Halpern, J. P. 1994, ApJS, 90, 1

Eracleous, M., \& Halpern, J. P. 2003, ApJ, 599, 886

Eracleous, M., Halpern, J. P., Gilbert, A. M., Newman, J. A., \& Filippenko, A. V. 1997, ApJ, 490, 216

Eracleous, M., Halpern, J. P., \& Livio, M. 1996, ApJ, 459, 89

Eracleous, M., Lewis, K. T., \& Flohic, H. M. L. G. 2009, New Astr. Rev., 53, 133

Escala, A., Larson, R. B., Coppi, P. S., \& Mardones, D. 2004, ApJ, 607, 765

Gaskell, C. M. 1983, in Proc. of Liége International Astrophysical Colloquia, Vol. 24, Quasars and Gravitational Lenses , ed. J.-P. Swings (Cointe-Ougreée Univ. Liége)), 473-477

Gaskell, C. M. 1996, ApJ, 464, L107

Gezari, S., Halpern, J. P., \& Eracleous, M. 2007, ApJS, 169, 167

Grier, C. J., Peterson, B. M., Pogge, R. W., et al. 2012, ApJ, 755, 60

Halpern, J. P., Eracleous, M., Filippenko, A. V., \& Chen, K. 1996, ApJ, 464, 704

Hayasaki, K., Mineshige, S., \& Sudou, H. 2007, PASJ, 59, 427

Holley-Bockelmann, K. \& Khan, F. M. 2015, ApJ, 810, 139

Hopkins, P. F., Hernquist, L., Cox, T. J., et al. 2006, ApJS, 163, 1

Khan, F. M., Holley-Bockelmann, K., Berczik, P., \& Just, A. 2013, ApJ, 773, 100

Lampton, M., Margon, B., \& Bowyer, S. 1976, ApJ, 208, 177

Lehto, H. J., \& Valtonen, M. J. 1996, ApJ, 460, 207

Newman, J. A., Eracleous, M., Filippenko, A. V. \& Halpern, J. P. 1997, ApJ, 485, 570

Valtonen, M. J., Lehto, H. J., Sillanpää, A., Nilsson, K., Mikkola, S., Hudec, R., Basta, M., Teräsranta, H., Haque, S., \& Rampadarath, H. 2006, ApJ, 646, 36

Lewis, K. T., \& Eracleous, M. 2006, ApJ, 642, 711

Lewis, K. T., Eracleous, M., \& Storchi-Bergmann, T. 2010, ApJS, 187,416

Liu, X., Shen, Y., Bian, F., Loeb, A., \& Tremaine, S. 2014, ApJ, 789,140

Lodato, G., Nayakshin, S., King, A. R., \& Pringle, J. E. 2009, MNRAS, 398, 1392

Lomb, N. R. 1976, Ap\&SS, 39, 447

Maness, H. L., Taylor, G. B., Zavala, R. T., Peck, A. B., \& Pollack, L. K. 2004, ApJ, 602, 123
Merritt, D., \& Ekers, R. D. 2002, Science, 297, 1310

Merritt, D., \& Poon, M. Y. 2004, ApJ, 606, 788

Netzer, H. 1982, MNRAS, 198, 589

Osterbrock, D. E., Koski, A. T., \& Phillips, M. M. 1976, ApJ, 206, 898

Popović, L. Č. 2012, New Astr. Rev., 56, 74

Popović, L. C., Shapovalova, A. I., Ilić, D., et al. 2014, A\&A, 572, A66

Quinlan, G. D. 1996, New A. R., 1, 35

Rodriguez, C., Taylor, G. B., Zavala, R. T., et al. 2006, ApJ, 646, 49

Rodriguez, C., Taylor, G. B., Zavala, R. T., Pihlström, Y. M., \& Peck, A. B. 2009, ApJ, 697, 37

Scargle, J. D. 1982, ApJ, 263, 835

Schimoia, J. S., Storchi-Bergmann, T., Grupe, D., et al. 2015, ApJ, 800, 63

Schnittman, J. D. 2013, Classical and Quantum Gravity, 30, 244007

Sergeev, S. G., Klimanov, S. A., Doroshenko, V. T., et al. 2011, MNRAS, 410, 1877

Sesana, A., Haardt, F., \& Madau, P. 2006, ApJ, 651, 392

Shafer, R., Ward, M., \& Barr, P. 1985, Space Sci. Rev., 40, 637

Shapovalova, A. I., Burenkov, A. N., Carrasco, L., et al. 2001, A\&A, 376, 775

Shapovalova, A. I., Popović, L. Č., Burenkov, A. N., et al. 2013, A\&A, 559, A10

Shen, Y., Liu, X., Loeb, A., \& Tremaine, S. 2013, ApJ, 775, 49

Shen, Y., \& Loeb, A. 2010, ApJ, 725, 249

Sillanpaa, A., Haarala, S., Valtonen, M. J., Sundelius, B., \& Byrd, G. G. 1988, ApJ, 325, 628

Strateva, I. V., Strauss, M. A., Hao, L., et al. 2003, AJ, 126, 1720

Tsalmantza, P., Decarli, R., Dotti, M., \& Hogg, D. W. 2011, ApJ, 738,20

Vasiliev, E., Antonini, F., \& Merritt, D. 2015, ApJ, 810, 49

Volonteri, M., Haardt, F., \& Madau, P. 2003, ApJ, 582, 559

Wang, L., Berczik, P., Spurzem, R., \& Kouwenhoven, M. B. N. 2014, ApJ, 780, 164

Yee, H. K. C., \& Oke, J. B. 1981, ApJ, 248, 472

Yu, Q. 2002, MNRAS, 331, 935

Zier, C., \& Biermann, P. L. 2002, A\&A, 396, 91 